\title{
Effect of polycations used in multi-walled carbon nanotubes thin films prepared by layer-by-layer technique on flexibility and gas sensing properties
}

\author{
Pi-Guey Su, Chuen-Chi Shiu, Chi-Ting Lee \\ Department of Chemistry, Chinese Culture University, Taipei 111, Taiwan, \\ spg@faculty.pccu.edu.tw
}

\begin{abstract}
:
In the current work, layer-by-layer self-assembled surface-oxidized multi-walled nanotubes (MWCNTs) and cationic polyelectrolyte (poly(allylamine hydrochloride)) (PAH) or poly(diallyldimethylammonium chloride) (PDDA) were synthesized on a polyester (PET) substrate for sensing $\mathrm{NH}_{3}$ gas. The electrical and gas sensing properties (response) and flexibility characteristics of the fabricated sensors depended strongly on the amounts of adsorbed MWCNTs and polycations type. The structural configuration of MWCNTs network in MWCNTs/PDDA multilayered thin film was more compact than that of MWCNTs/PAH. The conductivity of the MWCNTs/PDDA multilayered thin film was much higher than that of MWCNTs/PAH. The MWCNTs/PAH multilayered thin film showed a higher response and flexibility than that of MWCNTs/PDDA.
\end{abstract}

Key words: layer-by-layer self-assembled, multi-walled nanotubes, flexibility, polycations.

\section{Introduction:}

Fabrication of organic electronic devices on plastic substrates has attracted much interest recently, because of the proliferation of handheld portable consumer electronics. A new trend towards the direct integration of sensors on flexible substrates has become evident. The development of sensors should be extensible to the sensing of various gases. The main challenge is not only their manufacture, but also the stability of their mechanical, electrical and gas-sensing properties. Carbon nanotubes (CNTs) with excellent mechanical properties which are associated with their high specific surface area and nano-scale structure that provides many sites where gases can react, constitute a class of promising building blocks for fabricating flexible chemical sensors on plastic substrates. Many approaches for fabricating CNT films on flexible substrates have been proposed. They include line pattering method $[1,2]$, vacuum filtration method [3] and dry-transfer printing method [4,5]. The layer-by-layer (LBL) self-assembly of multilayer films based on sequential adsorptions of ionized polyelectrolytes and oppositely charged materials in aqueous solutions has been developed $[6,7]$. LBL self-assembly has many advantages over other methods, including simplicity, low-cost, low temperature of deposition, controllable thickness (from nanometers to micrometers) and the lack of any need for complex equipment. Therefore, the preparation of CNT multilayered films on flexible substrates using LBL self-assembly has been studied [8-10]. The CNT thin films can be built with alternating layers of negatively charged CNTs and polycations. In these systems, electrostatic interactions dominate, and it has been demonstrated that the film assembly and film properties are influenced by the charge density and conformation of the adsorbing species, and the ionic strength of the adsorption solutions [11]. Therefore, the flexibility, electrical and gas sensing properties of flexible chemical sensors markedly varies depending on the negatively charged CNTs and polycations. No attempt has been examined the electrical and gas sensing properties and flexibility of the assembled multi-walled carbon nanotubes (MWCNTs) multilayer thin films on a flexible substrate related to polycations used. In this study, two different types of MWCNTs multilayered thin films deposited on a flexible substrate were examined for sensing $\mathrm{NH}_{3}$ gas, in which poly(allylamine hydrochloride) (PAH) and poly(diallyldimethylammonium chloride) (PDDA) were used as cationic polymers and surface-oxidized MWCNTs as anionic species. The surface characteristics of the thin films were investigated in relation to polycations type by scanning electron microscopy (SEM). The effects of the polycations type on the electrical 
and gas sensing properties (response) and flexibility of the sensors were investigated.

\section{Experimental}

\subsection{Materials}

A dispersion of negatively charged MWCNTs dispersion was prepared using the well established acid treatment of MWCNTs that had been grown by raw chemical vapor deposition (CVD) (purity $>95 \%$, average diameter 20-30 nm, length $<50 \mu \mathrm{m}$, Sunnano Inc.) using an $\mathrm{H}_{2} \mathrm{SO}_{4} / \mathrm{HNO}_{3}$ mixture $(3: 1,50 \mathrm{ml})$, following by sonication at $80^{\circ} \mathrm{C}$ for $3 \mathrm{~h}$. The acid mixture was then decanted. The residue was then re-suspended in deionized water and centrifuged at $10,000 \mathrm{rpm}$ for $30 \mathrm{~min}$. This process was repeated many times until the solution $\mathrm{pH}$ was neutral, and was sonicated to facilitate a stable suspension of negatively charged MWCNTs in aqueous media. 3Mercapto-1-propanesulfonic acid sodium salt (MPS), anionic poly(4-styrenesulfonic acid-comaleic acid, SS:MA 1:1) sodium salt (PSSMA $1: 1, M w=20,000)$ and cationic poly(allylamine hydrochloride) (PAH; $\quad \mathrm{Mw}=15,000) \quad$ and poly(diallyldimethylammonium chloride) (PDDA; $M w=200,000-350,000)$ were obtained from Aldrich, the chemical structures of two cationic polymers were shown in Fig. 1.
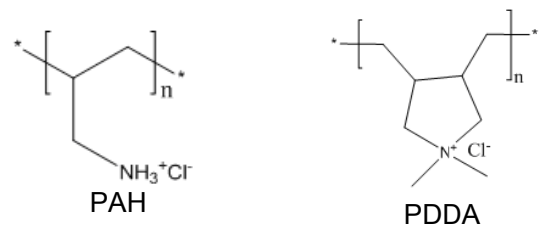

Fig. 1 Chemical structures of PAH and PDDA.

\subsection{Preparation of LBL MWCNTs multilayered thin films}

Figure 2(a) schematically depicts the structure of the flexible gas sensor. The interdigited gold electrodes (IDE) were made by sputtering $\mathrm{Cr}$ (50 nm thick) and then $\mathrm{Au}(250 \mathrm{~nm}$ thick) at temperature from 120 to $160{ }^{\circ} \mathrm{C}$. The gap between the electrodes was $0.25 \mathrm{~mm}$. The substrates were first immersed in a bath that contained a solution of $\mathrm{H}_{2} \mathrm{O}_{2} / \mathrm{H}_{2} \mathrm{SO}_{4}$ (1:2 volume ratio) for $3 \mathrm{~min}$. They were then thoroughly rinsed with DIW after each step. This process made the substrates hydrophilic. The negatively charged MPS/Au surface was prepared by immersing the hydrophilic Au/Cr/PET substrate in $2.0 \mathrm{mM}$ aqueous MPS for $24 \mathrm{~h}$, rinsing it with DIW and then drying it at $80^{\circ} \mathrm{C}$. A cyclic PSSMA/PAH bilayer film architecture was produced by alternately depositing aqueous $\mathrm{PAH}$ (0.06 mM at $\mathrm{pH}=4)$ and PSSMA (0.05 $\mathrm{mM}$ at $\mathrm{pH}=4$ ) onto the negatively charged MPS-modified substrate. For the production of each layer, the immersion time was about 10 min; which was followed by rinsing and drying. A two-cycle PSSMA/PAH bilayer film was fabricated by repeating the above processes, yielding a negatively charged (PSS/PAH) $)_{2} / \mathrm{MPS} / \mathrm{Au} / \mathrm{Cr} / \mathrm{PET}$ substrate. Then, an MWCNT multilayered thin film that was composed of (MWCNTs/PAH $)_{3}$ was deposited on the (PSSMA/PAH) $)_{2} / \mathrm{MPS} / \mathrm{Au} / \mathrm{Cr} / \mathrm{PET}$ substrate in the same manner as used to form the PSSMA/PAH multilayers. The optimal deposition time in MWCNTs active solution was for $20 \mathrm{~min}$. The (MWCNTs/PDDA) 3 multilayered thin film was deposited in the same manner as the MWCNTs/PAH multilayered thin film. Figure 2(b) shows the flexibility of the MWCNTs multilayered thin film assembled on a PET substrate. (a)

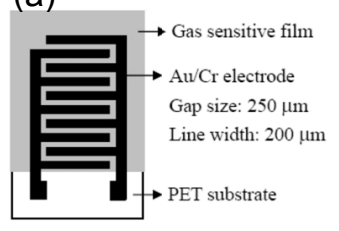

(b)

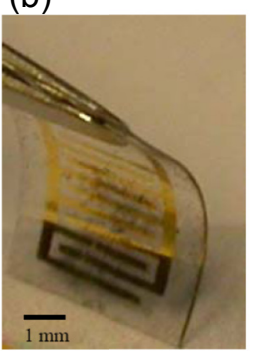

Fig. 2 (a) Structure of flexible gas sensor; (b) Photograph of bent gas sensor based on MWCNT multilayered thin film assembled on a PET substrate.

\subsection{Instruments and analysis}

The surface microstructure of the thin film that was coated on a PIET substrate was investigated using a field emission scanning electron microscope (FE-SEM, JEOL, JSM 6335F). The electrical and sensing characteristeristics were measured using a bench system at room temperature. Each sensor was connected in series with a load resistor and a fixed $5 \mathrm{~V}$ was continuously supplied to the sensor circuit from a power supply (GW, PST-3202). The desired $\mathrm{NH}_{3}$ gas concentrations, obtained by mixing a known volume of standard $\mathrm{NH}_{3}$ gas $(100,000 \mathrm{ppm})$, were injected into the chamber. Flexibility experiments were performed in which the sensors were bent to various degrees as their responses were monitored as a function of the period of exposure to $\mathrm{NH}_{3}$ gas. The bending angle was measured using a goniometer. The sensor response (S) was given by $S=\left(R_{\text {gas }}-\right.$ $R_{\text {air }} / R_{\text {air }}\left(\Delta R / R_{\text {air }}\right)$, where $R_{\text {gas }}$ and $R_{\text {air }}$ are the electrical resistances of the sensor in the tested gas and air, respectively.

\section{Results and discussion}

\subsection{Microstructure of surface of MWCNTs/PAH and MWCNTs/PDDA multilayered thin films}


Figure 4 shows the SEM image of the selfassembled of MWCNTs/PAH and MWCNTs/PDDA multilayer thin films on a modified PET substrate. The MWCNTs were randomly distributed on the surface and became entangled with each other to form a network in both MWCNTs/PAH and MWCNTs/PDDA multilayered thin films. The structural configuration of MWCNTs network in MWCNTs/PDDA multilayered thin film was more compact than that in MWCNTs/PAH, because the PDDA is a strong polycation. Therefore, to compensate for the opposite charge on the previously grown layer, more MWCNTs had to be adsorbed onto the surface, such that the amount of adsorbed MWCNTs in MWCNTs/PDDA multilayered thin film exceeded that of the MWCNTs/PAH.
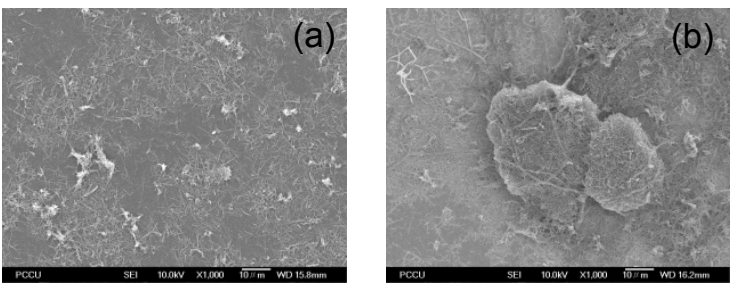

Fig. 4 FE-SEM images of (a): MWCNTS/PAH and (b): MWCNTS/PDDA multilayered thin films assembled on the

(PSSMA/PAH) 2 /MPS/Au/Cr/PET substrate

\subsection{Flexibility characteristics of of MWCNTs/PAH and MWCNTs/PDDA multilayered thin films}

Figure 5 plots the effect of polycations type used on the flexibility characteristics of the selfassembled of MWCNTs/polycations multilayer thin films. At each bending angle, the sensors were exposed to $50 \mathrm{ppm} \mathrm{NH}_{3}$ gas. The deviation of the responses of MWCNTs/PDDA multilayered thin film was higher than that of MWCNTs/PAH when the sensors were bent downward at an angle of up to $60^{\circ}$ from horizontal. Therefore, the MWCNTs/PAH multilayered thin film was more flexible than that of MWCNTs/PDDA. This result may be ascribed to the facts that the film structure of MWCNTs/polycation and the properties of used polycations. First, as described in Section 3.1, the film structure of MWCNTs/PDDA multilayered thin film was more compact (more rigid) than that of MWCNTs/PAH multilayered thin film, thus the flexibility of MWCNTs/PAH multilayered thin film was better than that of MWCNTs/PDDA multilayered thin film. Second, the $\mathrm{PAH}$ has a random branched structure, probably affording a flexible packed film. While a feature of PDDA is the ring structure of the diallyldimethylammonium residue (Fig. 1), which would result in the loss of flexibility of the polymer chain in the MWCNTs/polycation film.

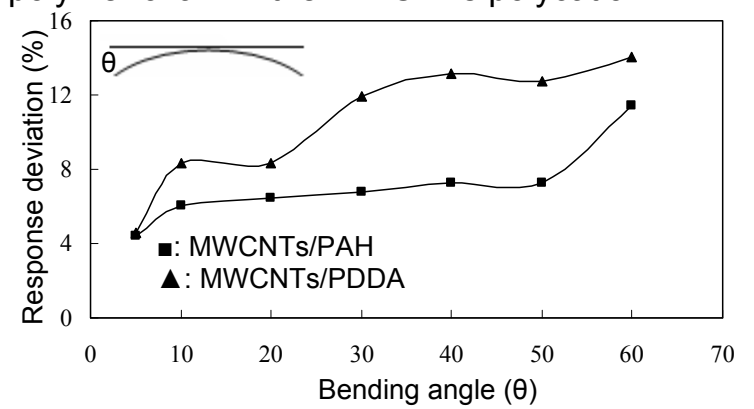

Fig. 5 Flexibility of MWCNTS/PAH and MWCNTS/PDDA multilayered thin film assembled on the (PSSMA/PAH) $2 / M P S / A u / C r / P E T$ substrate in response to $50 \mathrm{ppm} \mathrm{NH}$ gas.

\subsection{Gas sensing characteristics of of MWCNTs/PAH and MWCNTs/PDDA multilayered thin films}

The response of both PAH and PDDA multilayered thin films didn't have response to $\mathrm{NH}_{3}$ gas even the concentration of $\mathrm{NH}_{3}$ higher to $200 \mathrm{ppm}$, because the conductivity of the $\mathrm{PAH}$ and PDDA multilayered thin films both were much low. Therefore, in the MWCNTs/polycation multilayer film, it is considered that sensing of $\mathrm{NH}_{3}$ mainly occurs at the MWCNT layer. Figure 6 plots the responses of the MWCNTs/PAH and MWCNTs/PDDA multilayered thin films to various concentrations of $\mathrm{NH}_{3}$. The resistance of both MWCNTs/PAH and MWCNTs/PDDA multilayered thin films increased in presence of $\mathrm{NH}_{3}$ gas. The higher the $\mathrm{NH}_{3}$ gas concentration introduced, the more the resistance increased. The observed resistance increased when exposing the MWCNTs/polycations multilayered thin films to $\mathrm{NH}_{3}$ can be addressed to electron transfer from the $\mathrm{NH}_{3}$ to MWCNTs, that would lead to decreased hole carriers in the MWCNTs [12]. The base line resistance of both MWCNTs/PAH and MWCNTs/PDDA multilayered thin films was highly reproducible because that the $\mathrm{NH}_{3}$ adsorbed to MWCNTs is a physical adsorption $[13,14]$. The MWCNTs/PAH multilayered thin film showed higher response than that of MWCNTS/PDDA. It was expected that the employed MWCNTs/PDDA multilayered thin film should be promising for higher response than that of MWCNTs/PAH due to a larger content of MWCNTs in the MWCNTs/PDDA multilayered thin film assemblies than that of MWCNTs/PAH, inducing a much larger surface area and favoring the adsorption of gas molecules (as described in Section 3.1). On the contrary, the MWCNTs/PAH multilayered thin film showed higher response than that of MWCNTs/PDDA. This result may be ascribed to the fact that when the $\mathrm{NH}_{3}$ exposure to the employed highly conducting MWCNTs/PDDA 
multilayered thin film, however led to smaller changes in the charge carriers, therefore resulting in lower sensor response and, consequently, in lower response values [12]. Therefore, the sensor based on MWCNTs/PAH multilayered thin film was further tested for evaluation of other gas sensing characterizations. The flexible MWCNTs/PAH multilayered thin film sensor had good sensitivity and acceptable linearity $(\mathrm{Y}=$ $\left.0.0473 X+5.6689 ; R^{2}=0.9210\right)$ at the ranges in 10-200 ppm $\mathrm{NH}_{3}$ gas.

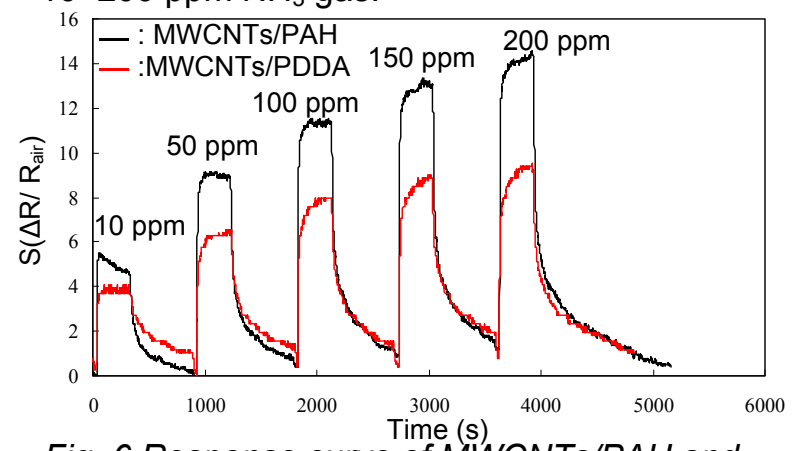

Fig. 6 Response curve of MWCNTs/PAH and MWCNTS/PDDA multilayered thin film as function of time (s) for various concentrations of $\mathrm{NH}_{3}$ gas.

\section{Conclusions:}

Novel flexible $\mathrm{NH}_{3}$ gas sensors which, can function at room temperature, were successfully fabricated by the layer-by-layer self-assembly of MWCNTs multilayer thin film a modified PET substrate using two different types of polycations (PAH and PDDA). The different response and flexibility between the MWCNTs/PAH and MWCNTs/PDDA multilayered thin films were relation to the different molecular geometry and flexibility of $\mathrm{PAH}$ and PDDA, which in turn result in the different MWCNTs/polycation film structures. The MWCNTS/PAH multilayered thin film showed a higher response and flexibility than that of MWCNTs/PDDA. The flexible MWCNTs/PAH multilayered thin film sensor had good sensitivity and acceptable linearity $(Y$ $=0.0473 X+5.6689 ; R^{2}=0.9210$ ) between 10 and $200 \mathrm{ppm}$. Due to simple fabricating process of this sensor, it would be suitable for use in a novel flexible $\mathrm{NH}_{3}$ gas sensor for future low-cost and flexible applications.

\section{Acknowledgements:}

The authors thank the National Science Council (Grant No. NSC 100-2113-M-034-001-MY3) of Taiwan for support.

\section{References:}

[1] K. Parikh, K. Cattanach, R. Rao, D. S. Suh, A. Wu, S. K. Manohar, Flexible vapor sensors using single walled carbon nanotubes, Sens. Actuators $B \quad 113$, 55-63 (2006); doi: 10.1016/ j.snb.2005.02.021.
[2] K. Cattanach, R. D. Kulkarni, M. Kozlov, S. K. Manohar, Flexible carbon nanotube sensors for nerve agent simulants, Nanotechnology 17, 4123-4128 (2006); doi: 10.1088/0957$4484 / 17 / 16 / 022$.

[3] C. S. Woo, C. H. Lim, C. W. Cho, B. Park, H. Ju, D. H. Min, C. J. Lee, S. B. Lee, Fabrication of flexible and transparent single-wall carbon nanotube gas sensors by vacuum filtration and poly(dimethyl siloxane) mold transfer, Microelectronic Engineering 84, 1610-1613 (2007); doi: 10.1016/j.mee.2007.01.162.

[4] Y. Sun, H. H. Wang, High-performance, flexible hydrogen sensors that use carbon nanotubes decorated with palladium nanoparticles, Adv. Mater. 19, 2818-2823 (2007); doi: 10.1002/adma.200602975.

[5] Y. Sun, H. H. Wang, M. Xia, Single-walled nanotubes modified with $\mathrm{Pd}$ nanoparticles: Unique building blocks for high-performance, flexible hydrogen sensors, J. Phys. Chem. C 112, 1250-1259 (2008); doi: 10.1021/jp076965n.

[6] G. Decher, J.D. Hong, J. Schmitt, Buildup of ultrathin multilayer films by a self-assembly process. III. Consecutively alternating adsorption of anionic and cationic polyelectrolytes on charged surface, Thin Solid Films 210, 831-835 (1992); doi: 10.1016/0040-6090(92)90417-A.

[7] G. Decher, Fuzzy nanoassemblies: toward layered polymeric multicomposites, Science 277 , 1232-1237 (1997); 10.1126/science.277.5330.1232

[8] X. B. Yan, X. J. Chen, B. K. Tay, K. A. Khor, Transparent and flexible glucose biosensor via layer-by-layer assembly of multi-wall carbon nanotubes and glucose oxidase, Electrochem. Commun. 9, 1269-1275 (2007); doi: 10.1016/j.elecom.2006.12.022.

[9] W. Xue, T. Cui, Electrical and electromechanical characteristics of self-assembled carbon nanotube thin films on flexible substrates, Sens. Actuators A 145-146, 330-335 (2008); doi: 10.1016/j.sna.2007.12.021.

[10] P. G. Su, Y. S. Chuang, Flexible $\mathrm{H}_{2}$ sensors fabricated by layer-by-layer self-assembly thin film of multi-walled carbon nanotubes and modified in situ with $\mathrm{Pd}$ nanoparticles, Sens. Actuators B 139, 488-493 (2010); doi:10.1016/j.snb.2009.03.051.

[11] E. Tjipto, J. E. Quinn, F. Caruso, Assembly of multilayer films from polyelectrolytes containing weak and strong acid moieties, Langmuir 21, 8785-8792 (2005); doi: 10.1021/la051197h.

[12] J. Kong, N. R. Franklin, C. Zhou, M. G. Chapline, S. Peng, K. Cho, H. Dai, Nanotube molecular wire as chemical sensors, Science 287, 622-625 (2000); doi: 10.1126/science.287.5453.622.

[13] H. Chang, J. D. Lee, S. M. Lee, Y. H. Lee, Adsorption of $\mathrm{NH}_{3}$ and $\mathrm{NO}_{2}$ molecules on carbon nanotubes, Appl. Phys. Lett. 79, 3863-3865 (2001); doi: 10.1063/1.1424069.

[14] K. Bradley, J. C. P. Gabriel, M. Briman, A. Star, G. Gruner, Charge transfer from ammonia physisorbed on nanotubes, Phys. Rev. Lett. 91, 218301/1-218301/4 (2003); doi: 10.1103/PhysRevLett.91.218301. 